\title{
Impedance Control and Modulation for Stable Footing in Locomotion of Biped Robots
}

\author{
Jong Hyeon Park and Hoam Chung \\ School of Mechanical Engineering \\ Hanyang University \\ Seoul, 133-791, Korea \\ Email: jongpark@email.hanyang.ac.kr
}

\begin{abstract}
This paper proposes an impedance control method with impedance modulation strategy for biped robot locomotion. Both the constrained leg and the free leg are controlled by impedance control. The impedance parameters changes depending on the gait phase of the biped robot. To reduce the magnitude of an impact and guarantee a stable footing when a foot contacts with the ground, the damping coefficient of the free leg is increased drastically and the reference trajectory of the leg is modified. Computer simulations with a 3DOF environment model for which a combination of a nonlinear and linear compliant contact models is used, show that the proposed control method works well and is superior to the computed-torque method in impact regulation.
\end{abstract}

\section{Introduction}

Implementations of biped robots that have high mobility in a tight living space of the human are a key to bringing more robots to the human. Currently, more researchers in many countries are working on biped robots than ever before.

Many different control laws for biped robots are proposed such as the computed-torque controller [1], the hybrid impedance/computed-torque controller [2], and the hybrid position/force controller [3]. While tracking the desired trajectory of the legs, a good biped robot controller should manage a stable contact of the swinging leg with the ground in its landing phase. Bouncing of the foot off the ground could cause instability in the locomotion.

In this paper, an impedance control with impedance modulation strategy is proposed to deal with ground contacts of the swinging leg. In typical human locomotion, leg muscles are repeatedly hardened and relaxed depending on the gait phase and result in very soft contacts with the ground. Using the same idea, the parameters of the impedance control are modulated depending on the its gait phase in order to have stable contacts.

To simulate locomotion of biped robots, the environment model must provide reasonably precise interaction forces. Plastic collision models such as the one proposed in [4] cannot simulate unstable contact transitions like foot bouncing that occurs when the foot with pads contacts with the ground.

In this paper, a 3-DOF environment model using the combination of nonlinear compliant contact model [5] and linear compliant model [6]. This model can simulate small movements of the feet due to the pads for shock absorption under the feet and provides realistic reaction forces.

The dynamics of the biped robot and 3-DOF environment model is described in Section 2. Design of the impedance controller and its impedance modulation strategy are presented in Section 3, followed by simulations in Section 4. Section 5 concludes.

\section{Models of Environment and Biped Robots}

\section{$2.1 \quad 3-D O F$ Environment Model}

The impact force can be very large when the foot of the freely swinging leg contacts with the ground. In order to control such impacts actively, robots should have controllers with very high bandwidth actuators with significantly large power. Instead, many biped robots are equipped with some kinds of shock- 


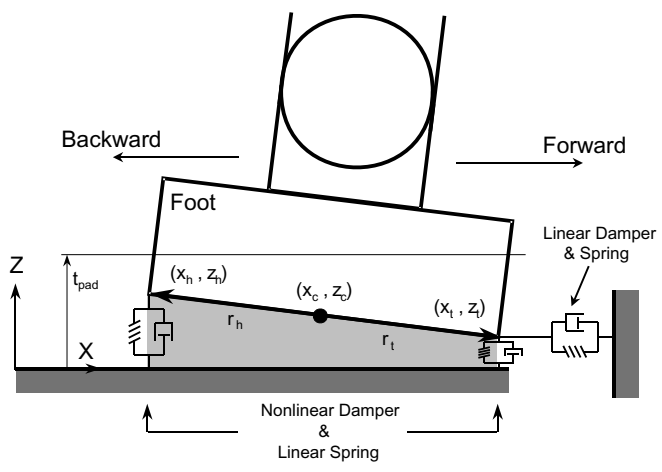

Figure 1: 3-DOF environment model

absorbing pads under their feet to avoid such large impacts. Such pads, in turn, allow small movements of the feet, and might cause unstable locomotion. In this paper, locomotion of biped robots with pads underneath their feet is considered. For simulation, pads are modeled as compliant contact models with linear springs and linear and nonlinear dampers. A nonlinear damper model is used in the vertical direction as [5] while a linear damper model is used in the horizontal direction, i.e., along the ground. The reaction force generated by the pad is thus

$$
f=-\frac{3}{2} \alpha k p \dot{p}-k p
$$

where $p$ is the penetration depth, $k$ is the stiffness, and $\alpha$ is a constant that defines the relation between the coefficient of restitution and the impact velocity.

Figure 1 describes a foot of the biped robot with a pad along with its environment, the ground. In the figure, $\left(x_{c}, z_{c}\right),\left(x_{h}, z_{h}\right)$, and $\left(x_{t}, z_{t}\right)$ denote the positions of the center of the sole, the heel and the toe of the foot, respectively; $r_{h}$ and $r_{t}$ denote vectors from $\left(x_{c}, z_{c}\right)$ to $\left(x_{h}, z_{h}\right)$, and from $\left(x_{c}, z_{c}\right)$ to $\left(x_{t}, z_{t}\right)$, respectively; and $t_{\text {pad }}$ denotes the thickness of the undeformed foot pad. Two sets of a nonlinear damper and a linear spring are located at the tip and heel of the foot to depict the pad effect in the vertical direction. A single set of a linear damper and spring model is located at the tip to handle the pad effect along the ground.

Thus, vertical force $f_{v}$ applied to the foot consists of the vertical forces at the heel and the toe, $f_{v, h}$ and

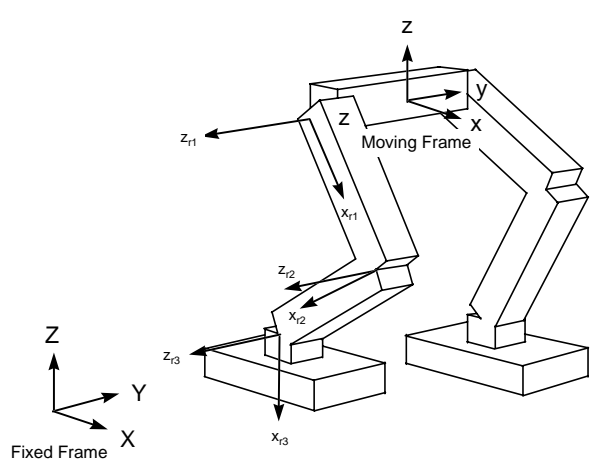

Figure 2: Coordinate frames of the biped robot

$f_{v, t}$, respectively, which are

$f_{v, i}= \begin{cases}-\frac{3}{2} \alpha k_{v}\left(t_{\text {pad }}-z_{i}\right) \dot{z}_{i}-k_{v}\left(t_{\text {pad }}-z_{i}\right) & z_{i}<t_{\text {pad }} \\ 0 & z_{i} \geq t_{\text {pad }}\end{cases}$

where $i=h$ for the heel, and $i=t$ for the tip.

$$
f_{v}=f_{v, h}+f_{v, t}
$$

For horizontal forces $f_{h}$,

$$
f_{h}=-b_{h} \dot{x}_{u e}-k_{h}\left(x_{u e}-x_{\text {org }}\right)
$$

where $x_{\text {org }}$ is the horizontal datum of the foot position to compute the horizontal elastic force, and is defined to be the position of the foot at the moment of its initial contact with the round. Assuming that the angle of the foot against the ground is negligible, we can neglect the moment due to the horizontal force. Thus, the moment $m_{c}$ exerted at $\left(x_{c}, z_{c}\right)$ is computed by

$$
m_{c}=r_{h} \times f_{v, h}+r_{c} \times f_{v, t},
$$

\subsection{Dynamics of Biped Robot}

The biped robot used in the paper is shown in Figure 2 , which has a 3 -DOF in each of its legs. Motions in the sagittal plane are considered here.

Biped robots are different from the typical manipulators in that they have no fixed contact points with the ground, and the constraints between the feet and the ground change repeatedly as they walk. Depending on whether a leg is moving freely in space or is supporting the robot weight, a leg is called a "unconstrained" or "constrained" leg. The dynamics of the 
biped robot used in the paper is described by three equations: one for each of the unconstrained and constrained legs and one for describing hip the dynamics and the action/reaction force between the hip and the legs. Thus,

$$
\begin{gathered}
H_{c} \ddot{q}_{c}+G_{c} a_{0}+D_{c} h_{c}+n_{c}=\tau_{c} \\
H_{u} \ddot{q}_{u}+G_{u} a_{0}+n_{u}=\tau_{u} \\
Q_{c} \ddot{q}_{c}+Q_{u} \ddot{q}_{u}+R a_{0}+P_{c} h_{c}+g=0
\end{gathered}
$$

where $\ddot{q} \in \mathbb{R}^{6}, a_{0} \in \mathbb{R}^{6}$, and $h \in \mathbb{R}^{6}$ are the joint acceleration, acceleration of the base link, and constrained force, respectively; $H, G, D \in \mathbb{R}^{6 \times 6}$ are inertia matrix of the leg chain, the matrix which denotes the dynamic effects of the base link to each link chain, and a Jacobian, respectively; $n \in \mathbb{R}^{6}, Q, P, R \in \mathbb{R}^{6 \times 6}$, and $g \in \mathbb{R}^{6}$ are Coriolis and centripetal term, gravitational effects, the matrix denoting the dynamic effects of the link chains to the base link, the matrix denoting the dynamic effects of the constrained force to the base link, the inertia matrix of the base link, and a term including the gravitational effects of the base link.

Equations (5) and (6) describe the dynamics of the supporting and free legs, respectively, and Eq. (7) describes the dynamics of the base link coupled with the legs. Subscripts 'c' and 'u' denotes 'constrained' and 'unconstrained', respectively. Note that the external force term should be inserted to the Eq. (6) in the weight acceptance phases.

\section{Impedance Control of Biped Robot}

The control law is derived under the assumption that there exist contact sensors and force sensors at the robot feet and that the controller knows when a foot is in contact with the ground as in [2].

\subsection{Gait Phases and Control Strategy}

According to an orthopedic research on the gait of normal human walking, each gait cycle has three main phases [7]: heel contact phase, stance phase, and swing phase, in that order. Moreover, the stance phase can be further divided into the weight acceptance phase and the single limb support phase.

Using some of the concept of the gait cycle, we divided a gait cycle into three phases depending on the load exerted at the leg: swing phase, weight acceptance phase, and single support phase. The leg moves freely in the space in the swing phase. The weight acceptance phase begins when the leg hits the ground, and ends when the other leg starts its swing phase.
During this phase, the leg should absorb the impact energy and accept the some of the robot weight. The single support phase begins at the end of weight acceptance phase, during which the leg supports the most of the robot weight and makes sure that the base link continues to moves forward.

This paper proposes to use different impedance models for legs depending on their phases. First, during the swing phase and the weight acceptance phase, the impedance model with respect to the foot is used. The only difference in them is that higher damping ratio is used in the weight acceptance phase in order to absorb the impact energy. During the single support phase, the constant impedance model with respect to the base link which is set for the trajectory tracking of base link is used to make sure that the base link moves more precisely.

\subsection{Impedance Control for Unconstrain- ed Leg}

From the relationship between the joint angular velocity, $\dot{q}$, and the foot velocity of the unconstrained leg, $\dot{x}_{u e} \in \mathbb{R}^{6}$,

$$
\dot{x}_{u e}=v_{0}+J_{u e} \dot{q}_{u}
$$

where $v_{0} \in \mathbb{R}^{6}$ is the velocity of the base link. Differentiating Eq. (8) results in

$$
\ddot{x}_{u e}=a_{0}+\dot{J}_{u e} \dot{q}_{u}+J_{u e} \ddot{q}_{u}
$$

or

$$
\ddot{q}_{u}=J_{u e}^{-1}\left(\ddot{x}_{u e}-a_{0}-\dot{J}_{u e} \dot{q}_{u}\right)
$$

Substituting this equation into Eq. (6) to replace $\ddot{q}_{u}$,

$$
\tau_{u}=H_{u} J_{u e}^{-1}\left(\ddot{x}_{u e}-a_{0}-\dot{J}_{u e} \dot{q}_{u}\right)+G_{u} a_{0}+n_{u}
$$

Suppose that the desired impedance of the unconstrained leg at its foot is

$$
\begin{aligned}
M_{u}\left(\ddot{x}_{u e}-\ddot{x}_{u e, d}\right)+B_{u}\left(\dot{x}_{u e}-\dot{x}_{u e, d}\right)+ & K_{u}\left(x_{u e}-x_{u e, d}\right) \\
& =f_{0}-f,
\end{aligned}
$$

where subscript 'd' denotes the desired value, $M_{u}, B_{u}$, and $K_{u}$ are the desired mass, damping ratio, and stiffness; and $f$ is the resultant external force. Due to the effect of the pad stiffness and the weight which should be transferred to weight accepting leg, reference force 
$f_{0}$ is expressed as

$$
f_{0}=\left\{\begin{array}{l}
{\left[\begin{array}{c}
0_{2 \times 1} \\
K_{u} t_{\text {pad }}+w_{t} \\
0_{3 \times 1}
\end{array}\right]} \\
0_{6 \times 1} \text { if the pad is squeezed } \\
\text { otherwise }
\end{array}\right.
$$

where $w_{t}$ is the target weight.

In order to achieve the desired impedance of Eq. (11), acceleration $\ddot{x}_{u e}$ should be

$$
\begin{aligned}
\ddot{x}_{u e}=\ddot{x}_{u e, d}-M_{u}^{-1} B_{u}\left(\dot{x}_{u e}-\dot{x}_{u e, d}\right) & \\
& -M_{u}^{-1} K_{u}\left(x_{u e}-x_{u e, d}\right)+M_{u}^{-1}\left(f_{0}-f\right),
\end{aligned}
$$

Assuming $\ddot{x}_{u e, d}=0$, and substituting Eq. (12) into Eq. (10) results in

$$
\begin{aligned}
\tau_{u}= & H_{u} J_{u e}^{-1}\left[-M_{u}^{-1} B_{u}\left(\dot{x}_{u e}-\dot{x}_{u e, d}\right)\right. \\
& -M_{u}^{-1} K_{u}\left(x_{u e}-x_{u e, d}\right)+M_{u}^{-1}\left(f_{0}-f\right)-a_{0} \\
& \left.-\dot{J}_{u e} \dot{q}_{u}\right]+G_{u} a_{0}+n_{u},
\end{aligned}
$$

which is the joint torque for the unconstrained leg to behavior with the desired impedance in Eq. (11).

\subsection{Impedance Control for Constrained Leg}

The joint torque, $\tau_{c}$ for the constrained (supporting) leg is computed using the impedance model for base link. The impedance control law with respect to the base link can be derived similarly to the method used in [2].

From Eq. (6) and Eq. (5)

$$
\begin{aligned}
& \ddot{q}_{c}=H_{c}^{-1}\left(\tau_{c}-G_{c} a_{0}-D_{c} h_{c}-n_{c}\right) \\
& \ddot{q}_{u}=H_{u}^{-1}\left(\tau_{u}-G_{u} a_{0}-n_{u}\right)
\end{aligned}
$$

Substituting Eqs. (14) and (15) into Eq. (7) results in

$$
Q_{c} H_{c}^{-1} \tau_{c}-\tilde{R} a_{0}+\tilde{P}_{c} h_{c}+\tilde{g}=0,
$$

where

$$
\begin{aligned}
\tilde{R} & =Q_{c} H_{c}^{-1} G_{c}+Q_{u} H_{u}^{-1} G_{u}-R \\
\tilde{g} & =g+Q_{u} H_{u}^{-1}\left(\tau_{u}-L_{u}\right)-Q_{c} H_{c}^{-1} n_{c} \\
\tilde{P} & =P_{c}-Q_{c} H_{u}^{-1} D_{c}
\end{aligned}
$$

Thus,

$$
\tau_{c}=\left(Q_{c} H_{c}^{-1}\right)^{-1}\left\{\tilde{R} a_{0}-\tilde{P} h_{c}-\tilde{g}\right\}
$$

Suppose the desired impedance of the base link at its center is

$$
\begin{aligned}
M_{0}\left(a_{0}-a_{0, d}\right)+B_{0}\left(\dot{x}_{0}\right. & \left.-\dot{x}_{0, d}\right) \\
& +K_{0}\left(x_{0}-x_{0, d}\right)=0,
\end{aligned}
$$

where subscript ' 0 ' denotes the base link, $M_{0}, B_{0}$, and $K_{0}$ are the desired mass, damping ratio, and stiffness.

In order to achieve the desired impedance of Eq. (18), acceleration of the base link $a_{0}$ should be

$$
a_{0}=a_{0, d}-M_{0}^{-1}\left\{B_{0}\left(\dot{x}_{0}-\dot{x}_{0, d}\right)+K_{0}\left(x_{0}-x_{0, d}\right)\right\},
$$

Substituting Eq. (19) into Eq. (17) gives

$$
\begin{array}{r}
\tau_{c}=\left(Q_{c} H_{c}^{-1}\right)^{-1}\left[\tilde { R } \left\{a_{0, d}-M_{0}^{-1}\left\{B_{0}\left(\dot{x}_{0}-\dot{x}_{0, d}\right)\right.\right.\right. \\
\left.\left.\left.+K_{0}\left(x_{0}-x_{0, d}\right)\right\}\right\}-\tilde{P} h_{c}-\tilde{g}\right]
\end{array}
$$

\subsection{Impedance Modulation}

During the swing phase and weight acceptance phase, the leg experiences a transition from a free space motion to a constrained motion. While a relatively good tracking performance is required in the swing phase, and behaviors for the impact regulation and weight acceptance are needed from the moment of an impact to just before the single support phase. Especially, at the moment of impact, the impact shock should be absorbed and the bouncing of the foot must be suppressed.

At the moment of the contact, the control law increases the damping ratio of the foot impedance 50 times the critical damping ratio. The stiffness and mass components in the impedance model is selected to match the desired stiffness and the cutoff frequency of the controller [8]. This method is very simple but highly efficient to regulate the impact transition. To enhance the capability of the controller in its impact regulation, the controller force the vertical position to $z_{c}-t_{\text {pad }}$ and the vertical velocity to zero right after the initial contact is detected regardless of the reference trajectory of the foot. The validity of this impedance modulation strategy is proven in the following computer simulation.

\section{Simulations}

The effectiveness of the impedance control with impedance modulation is to be shown in computer 


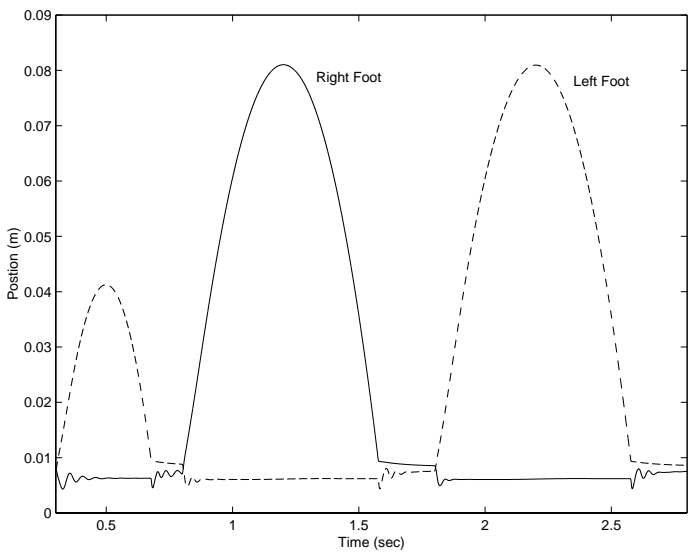

Figure 3: Walking with the hybrid control

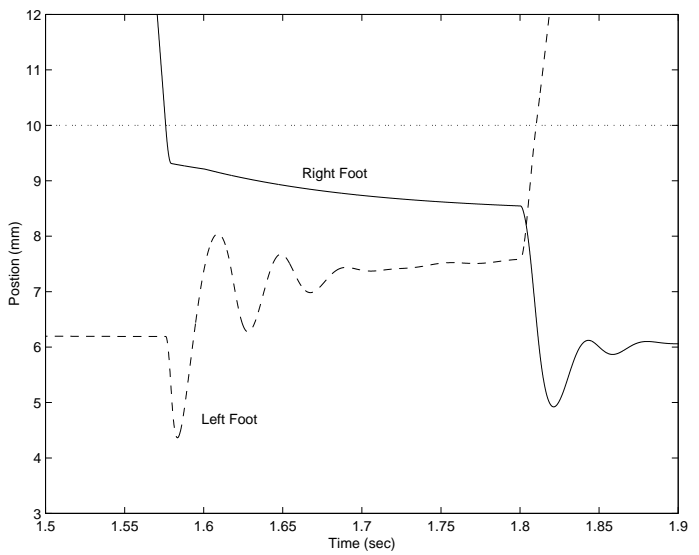

Figure 4: Closer view on the impact with the impedance control during the weight acceptance phase

simulations. The robot parameters for the simulations is summarized in Table 1. The parameters of the environment model used in the simulations is shown in Table 2. The pads underneath the feet are $1 \mathrm{~cm}$ thick.

In the first simulation, the proposed control algorithm is applied to the biped robot under the assumption that there is no uncertainty in the ground geometry. Initially, the robot stands still with its feet on the ground. Then the left leg is lifted first and the base link starts moving forward along the desired trajectory, which is based on the locomotion at $0.1 \mathrm{~m}$ in $0.4 \mathrm{~s}$. When the left leg comes into a contact with the ground, it moves into the weight acceptance phase for $0.1 \mathrm{~s}$.

From the second gait, the robot walks steadily and its gait parameters such as stance, maximum height of

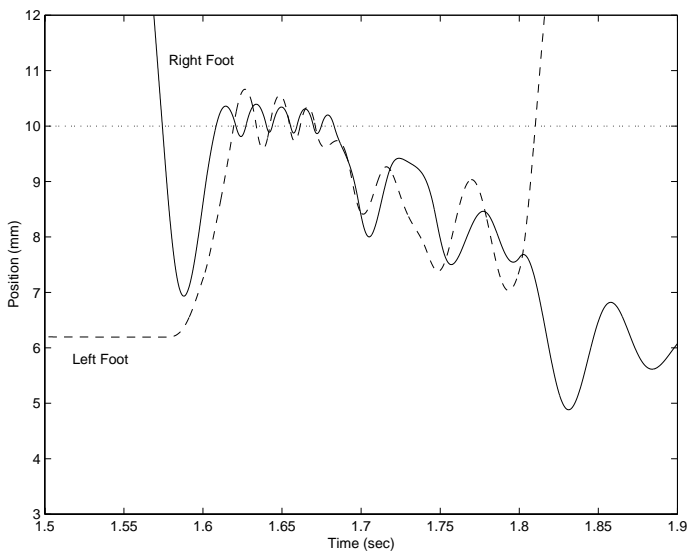

Figure 5: Impact with the computed-torque control method during the weight acceptance phase

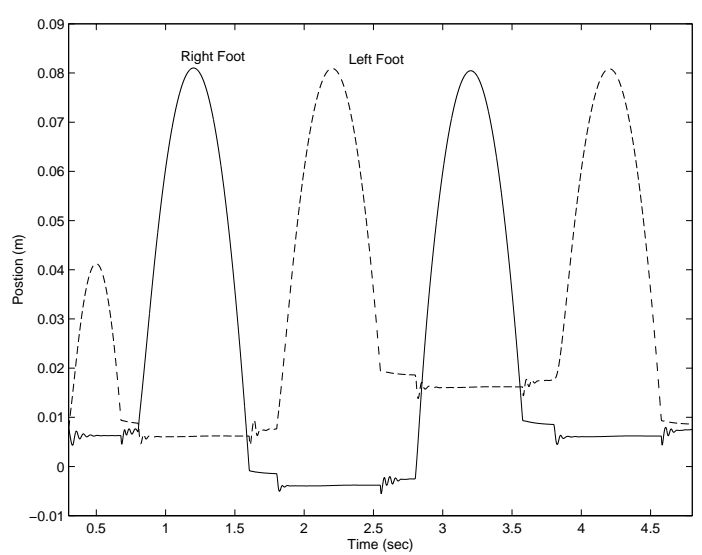

Figure 6: Walking on idealized uneven surface

foot and the period of gait become as twice as those in the first gait. Figure 3 shows the vertical motions of the feet. Figure 4 shows foot motions during the weight acceptance phase in more details. From this, it can be observed that the right foot does not bounce off the ground at the initial impact and increasingly takes more weight of the robot.

For comparisons, the same simulation but with only the computed-torque control method through out all the gait phases done. Its results are shown Figure 5. Note that the right foot bounces off the ground initially, and the entire supporting state become unstable.

According to [9], a small deviation of the ground level can cause the destabilization of the entire walking with position control only. In the second simula- 
Table 1: The parameters of the biped robot

\begin{tabular}{|c|c|c|}
\hline link & link length $(\mathrm{m})$ & link mass $(\mathrm{kg})$ \\
\hline 1 & 0.3 & 1 \\
\hline 2 & 0.3 & 1 \\
\hline 3 & 0.1 & 1 \\
\hline base & 0.3 & 10 \\
\hline
\end{tabular}

Table 2: The parameters of the environment model

\begin{tabular}{|c|c||c|c|}
\hline$\alpha$ & 0.5 & $k_{v}$ & $2.0 \times 10^{4} \mathrm{~N} / \mathrm{m}$ \\
\hline$k_{h}$ & $1.5 \times 10^{5} \mathrm{~N} / \mathrm{m}$ & $b_{h}$ & $1000 \mathrm{Ns} / \mathrm{m}$ \\
\hline
\end{tabular}

tion, response to the uncertainty in the ground level is simulated. At the second gait, the robot encounters the ground level that is $1 \mathrm{~cm}$ lower than the previous gait. The ground level becomes $2 \mathrm{~cm}$ higher than the second gait at the third gait. Figure 6 shows that the excellent adaptability of the proposed controller to an uneven ground surface, though the on-line adaptation used in the proposed control is only the calculation of $z_{c}$ for the reference trajectory modification at the start of weight acceptance phases.

The results of the simulations show that the proposed impedance controller regulates impact shocks and contacts with the ground very well and robustly.

\section{Conclusions}

An impedance controller with impedance modulation is proposed to control biped robots which repeatedly interact with the external environment. To investigate the performance of the proposed controller, biped robot locomotion is simulated with a 3-DOF environment model with compliant contact models. The performance of the proposed controller is compared with that of the computed-torque controller. The robustness of the proposed controller is verified through the simulations with ground level uncertainties. The simulation results shows that the proposed controller performs better than the computed-torque controller in stabilizing the robot footing.

\section{References}

[1] J. H. Park and K. D. Kim. Biped robot walking using gravity-compensated inverted pendulum mode and computed torque control. In IEEE Conf. on Robotics and Automation, Lueven, Belgium, 5 1998.

[2] J. H. Park and H. Chung. Hybrid control for biped robots using impedance control and computedtorque control. In IEEE Conf. on Robotics and Automation, Detroit, USA, 51999.

[3] Y. Fujimoto and A. Kawamura. Biped walking control with optimal foot force distribution by quadratic programing. In IEEE/ASME Conf. on Advanced Intelligent Mechatronics, Tokyo, Japan, 51997.

[4] Y. Fujimoto and A. Kawamura. Three dimensional digital simulation and autonomous walking control for eight-axis biped robot. In IEEE Conf. on Robotics and Automation, Nagoya, Japan, 51995.

[5] D. W. Marhefka and D. E. Orin. Simulation of contact using a nonlinear damping model. In IEEE Conf. on Robotics and Automation, Minneapolis, USA, 41996.

[6] P. R. Kraus and V. Kumar. Compliant contact models for rigid body collisions. In IEEE Conf. on Robotics and Automation, Albuquerque, USA, 41997.

[7] D. A. Winter. Overall principle of lower limb support during stance phase of gait. J. of Biomechanics, 13:123-127, 1980.

[8] H. Kazerooni, T. B. Sheridan, and P. K. Houpt. Robust compliant motion for manipulators, part i: The fundamental concepts of compliant motion. IEEE J. of Robotics and Automation, 2(2):83-92, 1986.

[9] J. Yamaguchi, A. Takanishi, and I. Kato. Experimental development of a foot mechanism with shock absorbing material for acquisition of landing surface position information and stabilization of dynamic biped walking. In IEEE Conf. on Robotics and Automation, Nagoya, Japan, 51995. 